\title{
Asymptotic zero distribution of Jacobi-Piñeiro and multiple Laguerre polynomials
}

\author{
Thorsten Neuschel, Walter Van Assche \\ Katholieke Universiteit Leuven, Belgium
}

August 15, 2018

\begin{abstract}
We give the asymptotic distribution of the zeros of Jacobi-Piñeiro polynomials and multiple Laguerre polynomials of the first kind. We use the nearest neighbor recurrence relations for these polynomials and a recent result on the ratio asymptotics of multiple orthogonal polynomials. We show how these asymptotic zero distributions are related to the Fuss-Catalan distribution.
\end{abstract}

\section{Introduction and main results}

In this paper we obtain the asymptotic distribution of the zeros of two families of multiple orthogonal polynomials: the Jacobi-Piñeiro polynomials and the multiple Laguerre polynomials of the first kind [9, Ch. 23], 2], 19]. These are two families of multiple orthogonal polynomials for which explicit formulas are known and which are useful for a number of applications. For instance, the zeros of Jacobi-Piñeiro polynomials (and Wronskian-type determinants of Jacobi-Piñeiro polynomials) form the unique solution of certain Bethe Ansatz equations [14] and multiple orthogonal polynomials are also useful for investigating determinantal point processes [10]. Recently the Jacobi-Piñeiro ensemble and the multiple Laguerre ensemble were introduced for random matrix minor processes related to percolation theory [1] which are based on the Jacobi-Piñeiro and multiple Laguerre polynomials of the first kind.

Let $\vec{n}=\left(n_{1}, n_{2}, \ldots, n_{r}\right) \in \mathbb{N}^{r}$ be a multi-index of size $|\vec{n}|=n_{1}+n_{2}+\cdots+n_{r}$. The Jacobi-Piñeiro polynomials $P_{\vec{n}}$, with parameters $\vec{\alpha}=\left(\alpha_{1}, \ldots, \alpha_{r}\right)$ and $\beta$, are type II multiple orthogonal polynomials on $[0,1]$ for $r$ Jacobi weights, i.e., $P_{\vec{n}}$ is a monic polynomial of degree $|\vec{n}|$ satisfying

$$
\int_{0}^{1} P_{\vec{n}}(x) x^{k} x^{\alpha_{j}}(1-x)^{\beta} d x=0, \quad k=0,1, \ldots, n_{j}-1,
$$

for $j=1,2, \ldots, r$, where $\beta>-1$ and $\alpha_{j}>-1$ for $1 \leq j \leq r$. They were introduced by Piñeiro for $\beta=0$ [17]. A multi-index $\vec{n}$ is normal if the monic multiple orthogonal polynomial $P_{\vec{n}}$ of degree $|\vec{n}|$ exists and is unique. All multi-indices for Jacobi-Piñeiro polynomials are normal when $\alpha_{i}-\alpha_{j} \notin \mathbb{Z}$ because then the measures form an AT-system 
[9, §23.1.2]. The polynomials are given by the Rodrigues formula

$$
\begin{aligned}
(-1)^{|\vec{n}|} \prod_{j=1}^{r}\left(|\vec{n}|+\alpha_{j}+\beta+1\right)_{n_{j}}(1-x)^{\beta} P_{\vec{n}}(x) & \\
& =\prod_{j=1}^{r}\left(x^{-\alpha_{j}} \frac{d^{n_{j}}}{d x^{n_{j}}} x^{n_{j}+\alpha_{j}}\right)(1-x)^{|\vec{n}|+\beta},
\end{aligned}
$$

where the product of the differential operators can be taken in any order, since these operators are commuting [9, §23.3.2]. Multiple Laguerre polynomials of the first kind $L_{\vec{n}}$ are given by the Rodrigues formula

$$
(-1)^{|\vec{n}|} e^{-x} L_{\vec{n}}(x)=\prod_{j=1}^{r}\left(x^{-\alpha_{j}} \frac{d^{n_{j}}}{d x^{n_{j}}} x^{n_{j}+\alpha_{j}}\right) e^{-x},
$$

where the product of the differential operators can be taken in any order [9, §23.4.1]. If the parameters $\vec{\alpha}=\left(\alpha_{1}, \alpha_{2}, \ldots, \alpha_{r}\right)$ are such that $\alpha_{i}>-1$ for every $i$ and $\alpha_{i}-\alpha_{j} \notin \mathbb{Z}$ $(1 \leq i, j \leq r)$, then all multi-indices are normal and the polynomials satisfy the following orthogonality properties

$$
\int_{0}^{\infty} L_{\vec{n}}(x) x^{k} x^{\alpha_{j}} e^{-x} d x=0, \quad k=0,1, \ldots, n_{j}-1,
$$

for $j=1,2, \ldots, r$. An explicit expression is given by

$$
\begin{aligned}
L_{\vec{n}}(x)= & \sum_{k_{1}=0}^{n_{1}} \cdots \sum_{k_{r}=0}^{n_{r}}(-1)^{|\vec{k}|} \frac{n_{1} !}{\left(n_{1}-k_{1}\right) !} \cdots \frac{n_{r} !}{\left(n_{r}-k_{r}\right) !} \\
& \times\left(\begin{array}{c}
n_{r}+\alpha_{r} \\
k_{r}
\end{array}\right)\left(\begin{array}{c}
n_{r}+n_{r-1}+\alpha_{r-1}-k_{r} \\
k_{r-1}
\end{array}\right) \cdots\left(\begin{array}{c}
|\vec{n}|-|\vec{k}|+k_{1}+\alpha_{1} \\
k_{1}
\end{array}\right) x^{|\vec{n}|-|\vec{k}|} .
\end{aligned}
$$

We will obtain the asymptotic distribution of the zeros of these multiple orthogonal polynomials by using a result on the asymptotic behavior of the ratio of two neighboring polynomials [21]. This result uses the nearest neighbor recurrence relations for multiple orthogonal polynomials

$$
x P_{\vec{n}}(x)=P_{\vec{n}+\vec{e}_{k}}(x)+b_{\vec{n}, k} P_{\vec{n}}(x)+\sum_{j=1}^{r} a_{\vec{n}, j} P_{\vec{n}-\vec{e}_{j}}(x), \quad 1 \leq k \leq r,
$$

where $\vec{e}_{j}=(0, \ldots, 0,1,0, \ldots, 0)$ with 1 in the $j$ th entry, and some knowledge about the asymptotic behavior for the recurrence coefficients $a_{\vec{n}, j}, b_{\vec{n}, j}(1 \leq j \leq r)$. The ratio asymptotic behavior for Jacobi-Piñeiro polynomials will be obtained in Section 2 and for multiple Laguerre polynomials of the first kind in Section 5. The asymptotic distribution of the zeros of Jacobi-Piñeiro polynomials will be obtained in Section 4, where the following result will be proved. We will use the multi-index $\overrightarrow{1}=(1,1, \ldots, 1)$ so that the diagonal index is $(n, n, \ldots, n)=n \overrightarrow{1}$. 
Theorem 1.1. Let $0<x_{1, r n}<x_{2, r n}<\cdots<x_{r n, r n}<1$ be the zeros of the Jacobi-Pineiro polynomial $P_{n \overrightarrow{1}}$ with multi-index $n \overrightarrow{1}=(n, n, \ldots, n)$. Then for every continuous function $f$ on $[0,1]$ one has

$$
\lim _{n \rightarrow \infty} \frac{1}{r n} \sum_{k=1}^{r n} f\left(x_{k, r n}\right)=\int_{0}^{1} f(t) v_{r}(t) d t
$$

where the density $v_{r}$ on $[0,1]$ is given by means of a density $w_{r}$ on $\left[0, c_{r}\right]$ as

$$
v_{r}(x)=c_{r} w_{r}\left(c_{r} x\right), \quad c_{r}=\frac{(r+1)^{r+1}}{r^{r}},
$$

and with the change of variables

$$
\hat{x}=c_{r} x=\frac{(\sin (r+1) \varphi)^{r+1}}{\sin \varphi(\sin r \varphi)^{r}}, \quad 0<\varphi<\frac{\pi}{r+1},
$$

the density $w_{r}$ is

$$
\begin{aligned}
w_{r}(\hat{x}) & =\frac{r+1}{\pi} \frac{1}{\left|\hat{x}^{\prime}(\varphi)\right|} \\
& =\frac{r+1}{\pi \hat{x}} \frac{\sin \varphi \sin r \varphi \sin (r+1) \varphi}{(r+1)^{2} \sin ^{2} r \varphi-2 r(r+1) \sin (r+1) \varphi \sin r \varphi \cos \varphi+r^{2} \sin ^{2}(r+1) \varphi} .
\end{aligned}
$$

The density $w_{r}$ is in fact the uniform density on $\left[0, \frac{\pi}{r+1}\right]$ in the variable $\varphi$ since

$$
\int_{0}^{c_{r}} f(\hat{x}) w_{r}(\hat{x}) d \hat{x}=\int_{0}^{\frac{\pi}{r+1}} f(\hat{x}(\varphi)) w_{r}(\hat{x}(\varphi))\left|\hat{x}^{\prime}(\varphi)\right| d \varphi=\frac{r+1}{\pi} \int_{0}^{\frac{\pi}{r+1}} f(\hat{x}(\varphi)) d \varphi .
$$

In this sense Theorem 1.1 is the extension to multiple orthogonal polynomials of the equidistribution result for zeros of orthogonal polynomials [18, Thm. 12.7.2] for the case of Jacobi-Piñeiro polynomials. In fact, the same asymptotic distribution of zeros will hold for all multiple orthogonal polynomials for which the nearest neighbor recurrence coefficients behave as in (2.3) $-(2.4)$, provided the zeros of neighboring polynomials interlace. We have plotted the density $v_{r}$ on $[0,1]$ for $1 \leq r \leq 5$ in Figure 1.

Observe that

$$
\hat{x}=c_{r}-\left(\begin{array}{c}
r+1 \\
2
\end{array}\right) c_{r} \varphi^{2}+\mathcal{O}\left(\varphi^{4}\right), \quad \varphi \rightarrow 0,
$$

and

$$
\hat{x}=\left(\frac{r+1}{\sin \frac{\pi}{r+1}}\right)^{r+1}\left(\frac{\pi}{r+1}-\varphi\right)^{r+1}+\mathcal{O}\left(\left(\frac{\pi}{r+1}-\varphi\right)^{r+2}\right), \quad \varphi \rightarrow \frac{\pi}{r+1},
$$

so that the density $v_{r}$ behaves as $\left(\hat{x}=c_{r} x\right)$

$$
v_{r}(x)=\mathcal{O}\left(\varphi^{-1}\right)=\mathcal{O}\left((1-x)^{-1 / 2}\right), \quad x \rightarrow 1,
$$

and

$$
v_{r}(x)=\mathcal{O}\left(\left(\frac{\pi}{r+1}-\varphi\right)^{-r}\right)=\mathcal{O}\left(x^{-\frac{r}{r+1}}\right), \quad x \rightarrow 0 .
$$




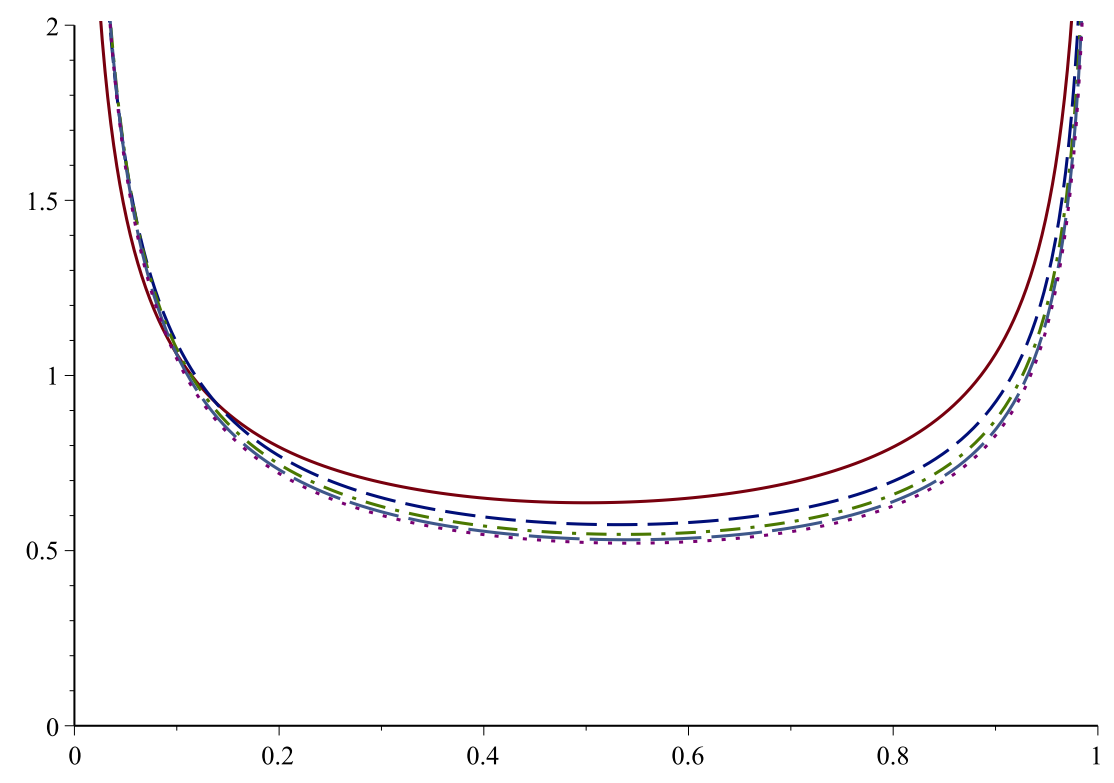

Figure 1: The asymptotic zero densities $v_{r}$ for Jacobi-Piñeiro polynomials: $r=1$ (solid), $r=2$ (dash), $r=3$ (dash dot), $r=4$ (long dash), and $r=5$ (dots).

Hence the densities $v_{r}$ have a square root singularity at 1 but a higher order singularity at 0 when $r>1$, which means that the zeros are more dense near the endpoints 0 and 1 , and even more so near 0 than near 1 when $r>1$. For $r=1$ the density $v_{1}$ is the well-known arcsin density on $[0,1]$,

$$
v_{1}(x)=\frac{1}{\pi} \frac{1}{\sqrt{x(1-x)}}, \quad 0<x<1
$$

which is the equilibrium measure for $[0,1]$ in logarithmic potential theory. For $r=2$ the density can explicitly be written as

$$
v_{2}(x)=\frac{\sqrt{3}}{4 \pi} \frac{(1+\sqrt{1-x})^{1 / 3}+(1-\sqrt{1-x})^{1 / 3}}{x^{2 / 3} \sqrt{1-x}}, \quad x \in(0,1),
$$

and this asymptotic zero distribution was already found in [3, Thm. 2.5]. The moments of $w_{r}$ are integers given by

$$
\int_{0}^{c_{r}} x^{n} w_{r}(x) d x=\frac{r+1}{\pi} \int_{0}^{\frac{\pi}{r+1}} x(\varphi)^{n} d \varphi=\left(\begin{array}{c}
(r+1) n \\
n
\end{array}\right), \quad n \in \mathbb{N}=\{0,1,2, \ldots\},
$$

which follows from [16, Remark 3.4].

For multiple Laguerre polynomials we need to use a scaling to prevent the zeros from going to infinity. The appropriate scaling is to divide all the zeros of $L_{\vec{n}}$ by $|\vec{n}|$, so that we are in fact investigating the zeros of $L_{n \overrightarrow{1}}(r n x)$ for the multi-index $n \overrightarrow{1}=(n, n, \ldots, n)$ on the diagonal. In Section 6 we obtain the asymptotic distribution of the scaled zeros, where we prove the following result. 
Theorem 1.2. Let $0<x_{1, r n}<x_{2, r n}<\cdots<x_{r n, r n}$ be the zeros of the multiple Laguerre polynomials $L_{n \overrightarrow{1}}$ with multi-index $n \overrightarrow{1}=(n, n, \ldots, n)$. Then for every continuous function $f$ on $\left[0, c_{r} / r\right]$ one has

$$
\lim _{n \rightarrow \infty} \frac{1}{r n} \sum_{k=1}^{r n} f\left(\frac{x_{k, r n}}{r n}\right)=\int_{0}^{c_{r}} f(t / r) u_{r}(t) d t, \quad c_{r}=\frac{(r+1)^{r+1}}{r^{r}},
$$

where the density $u_{r}$ on $\left[0, c_{r}\right]$ is given by

$$
u_{r}(\hat{x})=\frac{1}{r \pi} \frac{(\sin r \varphi)^{r+1}}{(\sin (r+1) \varphi)^{r}}, \quad 0<\varphi<\frac{\pi}{r+1},
$$

where

$$
\hat{x}=\frac{(\sin (r+1) \varphi)^{r+1}}{\sin \varphi(\sin r \varphi)^{r}}, \quad 0<\varphi<\frac{\pi}{r+1} .
$$

The densities $u_{r}$ for $1 \leq r \leq 5$ are plotted in Figure 2.

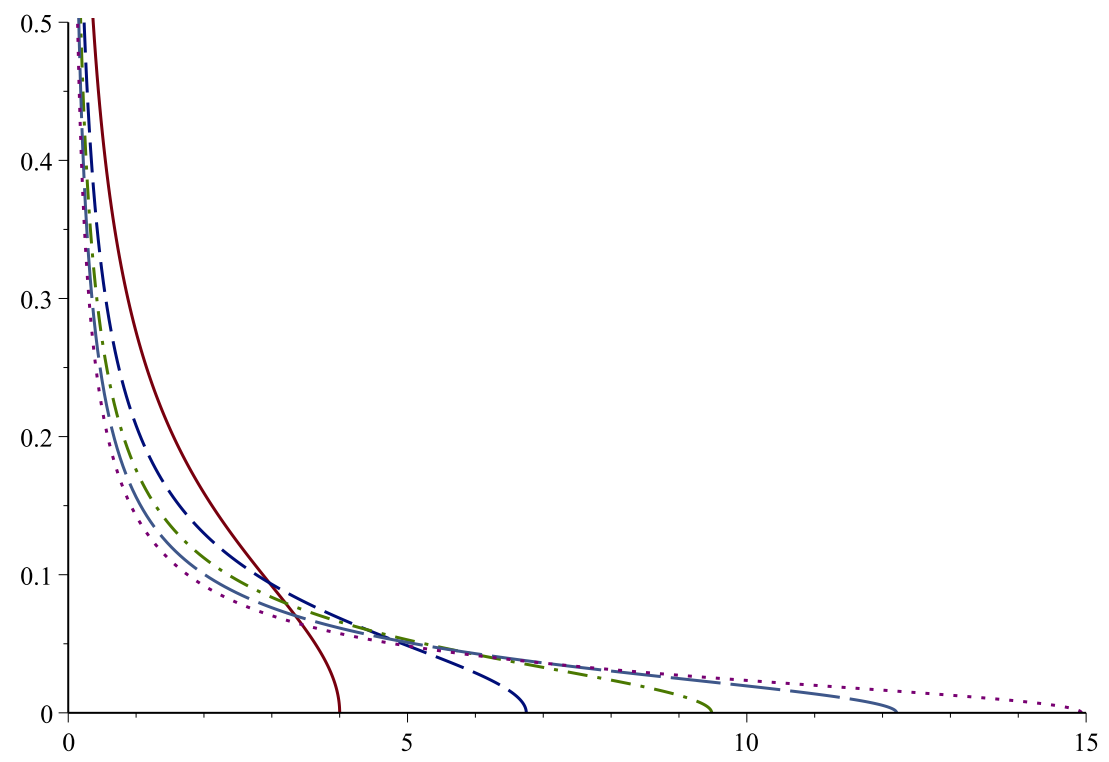

Figure 2: The asymptotic zero densities $u_{r}$ for multiple Laguerre polynomials of the first kind: $r=1$ (solid), $r=2$ (dash), $r=3$ (dash dot), $r=4$ (long dash), and $r=5$ (dots).

The density of the scaled zeros $\left\{\frac{x_{k, r n}}{r n}, 1 \leq k \leq r n\right\}$ is therefore given by $r u_{r}(r x)$ for $0<x<c_{r} / r$. Note that the densities $u_{r}$ behave as

$$
u_{r}(\hat{x})=\mathcal{O}(\varphi)=\mathcal{O}\left(\left(c_{r}-\hat{x}\right)^{1 / 2}\right), \quad \hat{x} \rightarrow c_{r},
$$

and

$$
u_{r}(\hat{x})=\mathcal{O}\left(\left(\frac{\pi}{r+1}-\varphi\right)^{-r}\right)=\mathcal{O}\left(\hat{x}^{-\frac{r}{r+1}}\right), \quad \hat{x} \rightarrow 0 .
$$

Hence the densities $u_{r}$ tend to zero as a square root near the endpoint $c_{r}$ and have the same singularity near 0 as in the Jacobi-Piñeiro case. For $r=1$ the density is the 
Marchenko-Pastur density [12]

$$
u_{1}(\hat{x})=\frac{1}{2 \pi} \sqrt{\frac{4-\hat{x}}{\hat{x}}}, \quad 0<\hat{x}<4
$$

which is also the known asymptotic distribution of the (scaled) zeros of Laguerre polynomials (see, e.g., [6]). For $r=2$ we have $u_{2}(\hat{x})=\frac{8}{27} g\left(\frac{8 \hat{x}}{27}\right)$, where

$$
g(y)=\frac{3 \sqrt{3}}{16 \pi} \frac{(1+3 \sqrt{1-y})(1-\sqrt{1-y})^{1 / 3}-(1-3 \sqrt{1-y})(1+\sqrt{1-y})^{1 / 3}}{y^{2 / 3}},
$$

and the asymptotic zero distribution of the zeros of multiple Laguerre polynomials for that case was already obtained in [3, Thm. 2.6]. An interesting observation is that the moments of $u_{r}$ are given by

$$
\int_{0}^{c_{r}} x^{n} u_{r}(x) d x=\frac{1}{n+1}\left(\begin{array}{c}
(r+1) n \\
n
\end{array}\right), \quad n \in \mathbb{N}
$$

The simple expressions for the moments of $w_{r}$ and $u_{r}$ on $\left[0, c_{r}\right]$ is the main reason why we prefer to express the asymptotic zero densities in terms of densities on $\left[0, c_{r}\right]$, rather than on $[0,1]$ and $\left[0, c_{r} / r\right]$ respectively. In Section 3 we will show that these densities and the asymptotic behavior of the ratio of Jacobi-Piñeiro and multiple Laguerre polynomials of the first kind are related to the Fuss-Catalan distribution with density

$$
g_{r}(x)=\frac{1}{\pi} \frac{\sin ^{2} \varphi(\sin r \varphi)^{r-1}}{(\sin (r+1) \varphi)^{r}}, \quad 0<x<c_{r}
$$

where

$$
x=\frac{(\sin (r+1) \varphi)^{r+1}}{\sin \varphi(\sin r \varphi)^{r}}, \quad 0<\varphi<\frac{\pi}{r+1},
$$

for which the moments are the Fuss-Catalan numbers [7, p. 347]

$$
\int_{0}^{c_{r}} x^{n} g_{r}(x) d x=\frac{1}{r n+1}\left(\begin{array}{c}
(r+1) n \\
n
\end{array}\right), \quad n \in \mathbb{N} .
$$

\section{Ratio asymptotics for Jacobi-Piñeiro polynomials}

The nearest neighbor recurrence relations are

$$
x P_{\vec{n}}(x)=P_{\vec{n}+\vec{e}_{k}}(x)+b_{\vec{n}, k} P_{\vec{n}}(x)+\sum_{j=1}^{r} a_{\vec{n}, j} P_{\vec{n}-\vec{e}_{j}}(x), \quad 1 \leq k \leq r,
$$

where the recurrence coefficients are given by

$$
\begin{aligned}
a_{\vec{n}, j}=\frac{n_{j}\left(n_{j}+\alpha_{j}\right)(|\vec{n}|+\beta)}{\left(|\vec{n}|+n_{j}+\right.} & \left.\alpha_{j}+\beta+1\right)\left(|\vec{n}|+n_{j}+\alpha_{j}+\beta\right)\left(|\vec{n}|+n_{j}+\alpha_{j}+\beta-1\right) \\
& \times \prod_{i=1}^{r} \frac{|\vec{n}|+\alpha_{i}+\beta}{|\vec{n}|+n_{i}+\alpha_{i}+\beta} \prod_{i=1, i \neq j}^{r} \frac{n_{j}+\alpha_{j}-\alpha_{i}}{n_{j}-n_{i}+\alpha_{j}-\alpha_{i}}, \quad 1 \leq j \leq r,
\end{aligned}
$$


and

$$
\begin{aligned}
& b_{\vec{n}, k}=(|\vec{n}|+\beta+1) \frac{\prod_{j=1}^{r}\left(|\vec{n}|+\beta+\alpha_{j}+1\right)}{\left(|\vec{n}|+n_{k}+\beta+\alpha_{k}+2\right) \prod_{j \neq k}\left(|\vec{n}|+n_{j}+\beta+\alpha_{j}+1\right)} \\
&-(|\vec{n}|+\beta) \frac{\prod_{j=1}^{r}\left(|\vec{n}|+\beta+\alpha_{j}\right)}{\prod_{j=1}^{r}\left(|\vec{n}|+n_{j}+\beta+\alpha_{j}\right)}, \quad 1 \leq k \leq r .
\end{aligned}
$$

(see, e.g., [20]).

If we take the multi-index $\vec{n}=\left(\left\lfloor q_{1} n\right\rfloor, \ldots,\left\lfloor q_{r} n\right\rfloor\right)$, where $q_{j}>0, \sum_{j=1}^{r} q_{j}=1$, and $\lfloor\cdot\rfloor$ is the floor function (i.e., $\lfloor a\rfloor=k$ whenever $k \leq a<k+1$ ), then the asymptotic behavior of the recurrence coefficients is

$$
\lim _{n \rightarrow \infty} a_{\vec{n}, j}=\frac{q_{j}^{r+1}}{\left(1+q_{j}\right)^{3}} \prod_{k=1}^{r} \frac{1}{1+q_{k}} \prod_{i \neq j} \frac{1}{q_{j}-q_{i}}=: a_{j}, \quad 1 \leq j \leq r,
$$

and with a bit of elementary calculus

$$
\lim _{n \rightarrow \infty} b_{\vec{n}, j}=\prod_{k=1}^{r} \frac{1}{1+q_{k}}\left(r+1-\sum_{k=1}^{r} \frac{1}{1+q_{k}}-\frac{1}{1+q_{j}}\right)=: b_{j}, \quad 1 \leq j \leq r .
$$

In order to have finite values of $a_{j}$, we assume for the moment that $q_{i} \neq q_{j}$ whenever $i \neq j$, but later on we will take the limit $q_{j} \rightarrow 1 / r$ for every $j$. This passage to the limit is allowed since the asymptotic distribution of the zeros is continuous in the parameters $\left(q_{1}, \ldots, q_{r}\right)$, which can be shown as in [4, Thm. 2]. We will use the notation

$$
p(\vec{q})=\prod_{k=1}^{r} \frac{1}{1+q_{k}}, \quad s=r+1-\sum_{k=1}^{r} \frac{1}{1+q_{k}}
$$

so that

$$
a_{j}=p(\vec{q}) \frac{q_{j}^{r+1}}{\left(1+q_{j}\right)^{3}} \prod_{i \neq j} \frac{1}{q_{j}-q_{i}}, \quad b_{j}=p(\vec{q})\left(s-\frac{1}{1+q_{j}}\right) .
$$

According to [21, Thm. 1.1], the ratio asymptotics for the Jacobi-Piñeiro polynomials with multi-index $\vec{n}=\left(\left\lfloor q_{1} n\right\rfloor, \ldots,\left\lfloor q_{r} n\right\rfloor\right)$ will then be given by

$$
\lim _{n \rightarrow \infty} \frac{P_{\vec{n}+\vec{e}_{k}}(x)}{P_{\vec{n}}(x)}=z(x)-b_{k}, \quad 1 \leq k \leq r,
$$

uniformly on compact subsets of $\mathbb{C} \backslash[0,1]$, where $z$ is the solution of the algebraic equation

$$
(z-x) B_{r}(z)+A_{r-1}(z)=0
$$

for which $z(x)-x \rightarrow 0$ when $x \rightarrow \infty$. In [21] the convergence was given uniformly on compact subsets of $\mathbb{C} \backslash \mathbb{R}$, but since all the zeros of Jacobi-Piñeiro polynomials are in $[0,1]$, the Stieltjes-Vitali theorem can be used to extend the uniform convergence to compact subsets of $\mathbb{C} \backslash[0,1]$. Here $B_{r}(z)=\prod_{j=1}^{r}\left(z-b_{j}\right)$ and $A_{r-1}$ is the polynomial of degree $r-1$ for which

$$
\frac{A_{r-1}(z)}{B_{r}(z)}=\sum_{j=1}^{r} \frac{a_{j}}{z-b_{j}}
$$


The latter means that the residue of $A_{r-1} / B_{r}$ at $b_{j}$ is given by $a_{j}$ :

$$
a_{j}=\frac{A_{r-1}\left(b_{j}\right)}{B_{r}^{\prime}\left(b_{j}\right)}=\frac{A_{r-1}\left(b_{j}\right)}{\prod_{i \neq j}\left(b_{j}-b_{i}\right)} .
$$

Observe that

$$
\prod_{i \neq j}\left(b_{j}-b_{i}\right)=\frac{p(\vec{q})^{r}}{\left(1+q_{j}\right)^{r-2}} \prod_{i \neq j}\left(q_{j}-q_{i}\right)
$$

so that the condition on the residues (2.8) becomes

$$
A_{r-1}\left(b_{j}\right)=\left(\frac{p(\vec{q}) q_{j}}{1+q_{j}}\right)^{r+1}, \quad 1 \leq j \leq r .
$$

This is a Lagrange interpolation problem. If we use (2.5) to write $q_{j}$ in terms of $b_{j}$, then

$$
q_{j}=\frac{1}{s-b_{j} / p(\vec{q})}-1
$$

so that

$$
\frac{p(\vec{q}) q_{j}}{1+q_{j}}=p(\vec{q})(1-s)+b_{j}, \quad 1 \leq j \leq r .
$$

The interpolation problem (2.9) then becomes

$$
A_{r-1}\left(b_{j}\right)=\left(b_{j}+p(\vec{q})(1-s)\right)^{r+1}, \quad 1 \leq j \leq r,
$$

hence $A_{r-1}(z)$ is a polynomial of degree $r-1$ interpolating the polynomial $(z+p(\vec{q})(1-$ $s))^{r+1}$ at the points $b_{j}(1 \leq j \leq r)$. If we take the limit where $q_{j} \rightarrow 1 / r$ for every $j$, then

$$
p(\vec{q}) \rightarrow\left(\frac{r}{r+1}\right)^{r}=: p, \quad s \rightarrow \frac{2 r+1}{r+1}, \quad b_{j} \rightarrow p\left(s-\frac{r}{r+1}\right)=p,
$$

hence all the interpolation points coincide. It is well known that the Lagrange interpolating polynomial for which all the interpolation points coincide corresponds to the Taylor polynomial of degree $r-1$ of the function $f(z)=(z+p(1-s))^{r+1}$ around the common interpolation point $p$. This Taylor polynomial of degree $r-1$ is the polynomial $(z+p(1-s))^{r+1}$ of degree $r+1$ from which we subtract the last two terms of the Taylor expansion around $p$ :

$$
\begin{aligned}
A_{r-1}(z) & =(z+p(1-s))^{r+1}-(z-p)^{r+1} \frac{f^{(r+1)}(p)}{(r+1) !}-(z-p)^{r} \frac{f^{(r)}(p)}{r !} \\
& =(z+p(1-s))^{r+1}-(z-p)^{r+1}-(r+1) p(2-s)(z-p)^{r} .
\end{aligned}
$$

The algebraic equation (2.7) for multi-indices on the diagonal then becomes

$$
(z-x)(z-p)^{r}+(z+p(1-s))^{r+1}-(z-p)^{r+1}-(r+1) p(2-s)(z-p)^{r}=0,
$$

which simplifies to

$$
x(z-p)^{r}=\left(z-\frac{p r}{r+1}\right)^{r+1} .
$$




\section{Relation with the Fuss-Catalan numbers}

Recently the Fuss-Catalan distribution and other related distributions (Raney distributions) appeared as limiting distributions of eigenvalues and singular values of certain random matrices [5], [15], [16]. In this section we will show how the ratio asymptotics in (2.6) is related to the Stieltjes transform of the Fuss-Catalan distribution. The weights $w_{r}$ and $u_{r}$ in Theorem 1.1 and 1.2 cannot be identified with the Fuss-Catalan distribution or any of the Raney distributions (except $u_{1}$, which is the Catalan distribution) because their behavior near the endpoints of the interval differs from the behavior of the Raney distributions given in 13 .

If we scale the variables $\hat{x}=c_{r} x$ and $\hat{z}=c_{r} z$, where

$$
c_{r}=\frac{(r+1)^{r+1}}{r^{r}}=\frac{r+1}{p},
$$

then the algebraic equation (2.11) becomes

$$
\hat{x}(\hat{z}-r-1)^{r}=(\hat{z}-r)^{r+1} .
$$

If we define

$$
\omega=\frac{\hat{z}-r}{\hat{z}-r-1}, \quad \hat{z}=\frac{(r+1) \omega-r}{\omega-1}
$$

then the algebraic equation becomes

$$
\omega^{r+1}+\hat{x}-\hat{x} \omega=0 .
$$

This is the algebraic equation for the generating function $G(1 / \hat{x})$ of the Fuss-Catalan numbers [7, p. 347] [16, Eq. (3.12)]. As in [16, §3], we assume that a solution exists of the form $\omega=\rho e^{i \varphi}$, where $\rho>0$ and $\varphi$ is real. Then inserting this in (3.3) gives

$$
\rho^{r+1} e^{i(r+1) \varphi}+\hat{x}-\hat{x} \rho e^{i \varphi}=0 .
$$

This gives for the real and the imaginary part

$$
\begin{aligned}
\rho^{r+1} \cos (r+1) \varphi+\hat{x}-\hat{x} \rho \cos \varphi & =0, \\
\rho^{r+1} \sin (r+1) \varphi-\hat{x} \rho \sin \varphi & =0 .
\end{aligned}
$$

From (3.5) we find

$$
\hat{x}=\rho^{r} \frac{\sin (r+1) \varphi}{\sin \varphi}
$$

and inserting this in (3.4) gives

$$
\rho(\hat{x})=\frac{\sin (r+1) \varphi}{\sin r \varphi}
$$

from which

$$
\hat{x}=\frac{(\sin (r+1) \varphi)^{r+1}}{\sin \varphi(\sin r \varphi)^{r}} .
$$

Observe that $\rho(x)>0$ for $0<\varphi<\frac{\pi}{r+1}$, and $\hat{x}$ is a monotonically decreasing function mapping $\left[0, \frac{\pi}{r+1}\right]$ into $\left[0, c_{r}\right]$. So for $\hat{x} \in\left[0, c_{r}\right]$ there is a solution $\rho e^{i \varphi}$ of the algebraic 
equation (3.3). The conjugate function $\rho e^{-i \varphi}$ is also a solution for $\hat{x} \in\left[0, c_{r}\right]$. In fact both solutions are the boundary value of a function $\omega$ which is analytic on $\mathbb{C} \backslash\left[0, c_{r}\right]$ and

$$
\omega_{+}=\lim _{\epsilon \rightarrow 0+} \omega(\hat{x}+i \epsilon)=\rho e^{-i \phi}, \quad \omega_{-}=\lim _{\epsilon \rightarrow 0+} \omega(\hat{x}-i \epsilon)=\rho e^{i \phi},
$$

because this $\omega$ is $G(1 / \hat{x})=\hat{x} F(\hat{x})$, where $F$ is the Stieltjes transform of the Fuss-Catalan distribution

$$
F(z)=\int_{0}^{c_{r}} \frac{g_{r}(y)}{z-y} d y, \quad z \in \mathbb{C} \backslash\left[0, c_{r}\right]
$$

with $g_{r}$ the Fuss-Catalan density, and a Stieltjes transform has the property that

$$
\operatorname{Im} F(z)\left\{\begin{array}{ll}
<0, & \operatorname{Im} z>0 \\
>0, & \operatorname{Im} z<0
\end{array} .\right.
$$

Observe that

$$
\frac{1}{z-p}=\lim _{n \rightarrow \infty} \frac{P_{\vec{n}}(x)}{P_{\vec{n}+\vec{e}_{k}}(x)}
$$

is the Stieltjes transform of a probability measure on $[0,1]$, since we have

$$
\frac{P_{\vec{n}}(x)}{P_{\vec{n}+\vec{e}_{k}}(x)}=\sum_{j=1}^{|\vec{n}|+1} \frac{c_{j, \vec{n}}}{x-x_{j, \vec{n}+\vec{e}_{k}}}
$$

and $c_{j, \vec{n}}>0$ because the zeros of $P_{\vec{n}}$ and $P_{\vec{n}+\vec{e}_{k}}$ interlace [8, Thm.2.1], and $\sum c_{j, \vec{n}}=1$ since we are dealing with monic polynomials. With the change of variables $\hat{x}=c_{r} x$ and $\hat{z}=c_{r} z$ it follows that

$$
1 /(\hat{z}-r-1)=\int_{0}^{c_{r}} \frac{d \mu(y)}{\hat{x}-y}
$$

is the Stieltjes transform of a probability distribution $\mu$ on $\left[0, c_{r}\right]$. Note that $(3.2)$ implies

$$
\frac{1}{\hat{z}-r-1}=\omega-1=\hat{x} F(\hat{x})-1
$$

where $F$ is the Stieltjes transform of the Fuss-Catalan distribution,

$$
F(\hat{x})=\frac{1}{\hat{x}} \sum_{n=0}^{\infty} \frac{1}{r n+1}\left(\begin{array}{c}
(r+1) n \\
n
\end{array}\right) \frac{1}{\hat{x}^{n}},
$$

so that $1 /(\hat{z}-r-1)$ is the Stieltjes transform of the probability measure for which the moments are the Fuss-Catalan numbers shifted by one

$$
\int_{0}^{c_{r}} y^{n} d \mu(y)=\frac{1}{r(n+1)+1}\left(\begin{array}{c}
(r+1)(n+1) \\
n+1
\end{array}\right),
$$

and hence this probability distribution has a density $\hat{x} g_{r}(\hat{x})$, where $g_{r}$ is the Fuss-Catalan density

$$
g_{r}(\hat{x})=\frac{1}{\pi} \frac{\sin ^{2} \varphi(\sin r \varphi)^{r-1}}{(\sin (r+1) \varphi)^{r}}, \quad 0<\varphi<\frac{\pi}{r+1}
$$


where $\hat{x}$ is given in (3.8). In particular this gives

$$
\frac{1}{\hat{z}-r-1}=\int_{0}^{c_{r}} \frac{y g_{r}(y)}{\hat{x}-y} d y .
$$

The weight is explicitly given by

$$
\hat{x} g_{r}(\hat{x})=\frac{1}{\pi} \frac{\sin \varphi \sin (r+1) \varphi}{\sin r \varphi}, \quad 0 \leq \varphi<\frac{\pi}{r+1},
$$

with $\hat{x}$ as in (3.8).

\section{Proof of Theorem 1.1}

So far we found that for $\vec{n}$ near the diagonal (i.e., $n_{j} / n \rightarrow 1 / r$ for every $j$ ) one has

$$
\lim _{n \rightarrow \infty} \frac{P_{\vec{n}+\vec{e}_{k}}(x)}{P_{\vec{n}}(x)}=z(x)-p=\frac{1}{c_{r}}(\hat{z}-r-1),
$$

uniformly for $x$ on compact subsets of $\mathbb{C} \backslash[0,1]$, or $\hat{x}$ on compact subsets of $\mathbb{C} \backslash\left[0, c_{r}\right]$. However we are interested in the asymptotic behavior of

$$
\frac{1}{|\vec{n}|} \frac{P_{\vec{n}}^{\prime}(x)}{P_{\vec{n}}(x)}
$$

where the prime' denotes the derivative with respect to $x$, because the limit will give the Stieltjes transform of the asymptotic distribution of the zeros of $P_{\vec{n}}$. By taking derivatives with respect to $x$ in (4.1) we find

$$
\lim _{n \rightarrow \infty} \frac{P_{\vec{n}+\vec{e}_{k}}(x)}{P_{\vec{n}}(x)}\left(\frac{P_{\vec{n}+\vec{e}_{k}}^{\prime}(x)}{P_{\vec{n}+\vec{e}_{k}}(x)}-\frac{P_{\vec{n}}^{\prime}(x)}{P_{\vec{n}}(x)}\right)=z^{\prime}=\frac{\hat{z}^{\prime}}{c_{r}},
$$

uniformly for $x$ on compact subsets of $\mathbb{C} \backslash[0,1]$. Together with (4.1), this gives

$$
\lim _{n \rightarrow \infty}\left(\frac{P_{\vec{n}+\vec{e}_{k}}^{\prime}(x)}{P_{\vec{n}+\vec{e}_{k}}(x)}-\frac{P_{\vec{n}}^{\prime}(x)}{P_{\vec{n}}(x)}\right)=\frac{\hat{z}^{\prime}}{\hat{z}-r-1} .
$$

If we use this result successively for each $k, 1 \leq k \leq r$, then we find for multi-indices on the diagonal $n \overrightarrow{1}=(n, n, \ldots, n)$ and $(n+1) \overrightarrow{1}=(n+1, n+1, \ldots, n+1)$

$$
\lim _{n \rightarrow \infty}\left(\frac{P_{(n+1) \overrightarrow{1}}^{\prime}(x)}{P_{(n+1) \overrightarrow{1}}(x)}-\frac{P_{n \overrightarrow{1}}^{\prime}(x)}{P_{n \overrightarrow{1}}(x)}\right)=\frac{r \hat{z}^{\prime}}{\hat{z}-r-1} .
$$

Then by taking averages (Cesàro's lemma) we get

$$
\lim _{n \rightarrow \infty} \frac{1}{n} \sum_{k=0}^{n-1}\left(\frac{P_{(k+1) \overrightarrow{1}}^{\prime}(x)}{P_{(k+1) \overrightarrow{1}}(x)}-\frac{P_{k \overrightarrow{1}}^{\prime}(x)}{P_{k \overrightarrow{1}}(x)}\right)=\frac{r \hat{z}^{\prime}}{\hat{z}-r-1},
$$

and since this contains a telescoping sum, this becomes

$$
\lim _{n \rightarrow \infty} \frac{1}{r n} \frac{P_{n \overrightarrow{1}}^{\prime}(x)}{P_{n \overrightarrow{1}}(x)}=\frac{\hat{z}^{\prime}}{\hat{z}-r-1},
$$


uniformly for $x$ on compact subsets of $\mathbb{C} \backslash[0,1]$, so that the right hand side is the Stieltjes transform of the asymptotic zero distribution of the zeros of $P_{n \overrightarrow{1}}$ for $n \overrightarrow{1}=(n, n, \ldots, n)$. From the Stieltjes transform we can find the density by using Stieltjes' inversion formula

$$
2 \pi i v_{r}(x)=\left(\frac{\hat{z}^{\prime}}{\hat{z}-r-1}\right)_{-}-\left(\frac{\hat{z}^{\prime}}{\hat{z}-r-1}\right)_{+} .
$$

Taking derivatives in (3.1) (and recalling that $\hat{x}=c_{r} x$ ) gives

$$
c_{r}(\hat{z}-r-1)^{r}+\hat{x} r(\hat{z}-r-1)^{r-1} \hat{z}^{\prime}=(r+1)(\hat{z}-r)^{r} \hat{z}^{\prime},
$$

so that

$$
\frac{\hat{z}^{\prime}}{\hat{z}-r-1}=\frac{c_{r}}{\hat{x}} \frac{\hat{z}-r}{\hat{z}-2 r-1} .
$$

Writing this in terms of $\omega$ using (3.2) gives

$$
\frac{\hat{z}^{\prime}}{\hat{z}-r-1}=\frac{c_{r}}{\hat{x}} \frac{\omega}{-r \omega+r+1} .
$$

Now use $\omega_{+}=\rho e^{-i \varphi}$ and $\omega_{-}=\rho e^{i \varphi}$ to find the density

$$
v_{r}(x)=\frac{r+1}{\pi x} \frac{\rho \sin \varphi}{\left|r \rho e^{i \varphi}-r-1\right|^{2}}
$$

and clearly $v_{r}(x)=v_{r}\left(\hat{x} / c_{r}\right)=c_{r} w_{r}(\hat{x})$, with the weight in (1.4). Observe that $\hat{x}$ : $\left[0, \frac{\pi}{r+1}\right] \rightarrow\left[0, c_{r}\right]$ is a monotonically decreasing function with

$$
\hat{x}^{\prime}(\varphi)=\frac{-\hat{x}}{\sin \varphi \sin r \varphi \sin (r+1) \varphi}\left|(r+1) \sin r \varphi-e^{i \varphi} r \sin (r+1) \varphi\right|^{2}
$$

so that

$$
w_{r}(\hat{x})=\frac{r+1}{\pi} \frac{1}{\left|\hat{x}^{\prime}(\varphi)\right|}, \quad 0<\varphi<\frac{\pi}{r+1}
$$

\section{Ratio asymptotics for multiple Laguerre polynomi- als of the first kind}

The nearest neighbor recurrence relations for multiple orthogonal polynomials of the first kind are given by

$$
x L_{\vec{n}}(x)=L_{\vec{n}+\vec{e}_{k}}(x)+b_{\vec{n}, k} L_{\vec{n}}(x)+\sum_{j=1}^{r} a_{\vec{n}, j} L_{\vec{n}-\vec{e}_{j}}(x), \quad 1 \leq k \leq r,
$$

where the recurrence coefficients are given by

$$
a_{\vec{n}, j}=n_{j}\left(n_{j}+\alpha_{j}\right) \prod_{i=1, i \neq j}^{r} \frac{n_{j}+\alpha_{j}-\alpha_{i}}{n_{j}-n_{i}+\alpha_{j}-\alpha_{i}}, \quad 1 \leq j \leq r
$$

and

$$
b_{\vec{n}, k}=|\vec{n}|+n_{k}+\alpha_{k}+1, \quad 1 \leq k \leq r .
$$


(see, e.g., [20]). We can now proceed as in the case of Jacobi-Piñeiro polynomials. The recurrence coefficients are somewhat easier but they are unbounded so that we need to use a scaling. Suppose again that $\vec{n}=\left(\left\lfloor q_{1} n\right\rfloor, \ldots,\left\lfloor q_{r} n\right\rfloor\right)$, where $q_{i} \neq q_{j}$ whenever $i \neq j$. It then follows that

$$
\lim _{n \rightarrow \infty} \frac{a_{\vec{n}, j}}{n^{2}}=q_{j}^{r+1} \prod_{i \neq j} \frac{1}{q_{j}-q_{i}}=: a_{j}, \quad 1 \leq j \leq r
$$

and

$$
\lim _{n \rightarrow \infty} \frac{b_{\vec{n}, j}}{n}=1+q_{j}=: b_{j}, \quad 1 \leq j \leq r .
$$

According to [21, Thm. 1.2] we then have

$$
\lim _{n \rightarrow \infty} \frac{L_{\vec{n}+\vec{e}_{k}}(n x)}{n L_{\vec{n}}(n x)}=z(x)-b_{k}, \quad 1 \leq k \leq r,
$$

uniformly on compact subsets of $\mathbb{C} \backslash[0, \infty)$, where $z$ is the solution of the algebraic equation

$$
(z-x) B_{r}(z)+A_{r-1}(z)=0
$$

where $B_{r}(z)=\prod_{j=1}^{r}\left(z-b_{j}\right)$ and $A_{r-1}$ is obtained from

$$
\frac{A_{r-1}(z)}{B_{r}(z)}=\sum_{j=1}^{r} \frac{a_{j}}{z-b_{j}} .
$$

The uniform convergence on compact subsets of $\mathbb{C} \backslash \mathbb{R}$ in $[21]$ can be extended to $\mathbb{C} \backslash[0, \infty)$ because the zeros of multiple Laguerre polynomials of the first kind are on $[0, \infty)$. One can even extend this further to $\mathbb{C} \backslash\left[0, c_{r} / r\right]$ since all the scaled zeros are dense on $\left[0, c_{r} / r\right]$, but we will not need this here. Observe that

$$
\prod_{i \neq j}\left(q_{j}-q_{i}\right)=\prod_{i \neq j}\left(b_{j}-b_{i}\right)
$$

so that we get the interpolation condition

$$
A_{r-1}\left(b_{j}\right)=q_{j}^{r+1}=\left(b_{j}-1\right)^{r+1}, \quad 1 \leq j \leq r .
$$

Hence $A_{r-1}(z)$ is the Lagrange interpolating polynomial of degree $r-1$ for the function $f(z)=(z-1)^{r+1}$ for the interpolation points $b_{1}, \ldots, b_{r}$. Now let $q_{j} \rightarrow 1 / r$ for every $j$, then

$$
b_{j} \rightarrow \frac{r+1}{r}
$$

and $A_{r-1}(z)$ will be the Taylor polynomial of degree $r-1$ around $\frac{r+1}{r}$ for the function $f(z)=(z-1)^{r+1}$. This gives

$$
A_{r-1}(z)=(z-1)^{r+1}-\left(z-\frac{r+1}{r}\right)^{r+1}-\frac{r+1}{r}\left(z-\frac{r+1}{r}\right)^{r} .
$$

The algebraic equation for multi-indices near the diagonal then becomes

$$
x\left(z-\frac{r+1}{r}\right)^{r}=(z-1)^{r+1} .
$$

The change of variables $r z=\hat{z}$ and $r x=\hat{x}$ gives the same algebraic equation as in (3.1). 


\section{Proof of Theorem 1.2}

As in Section 4 we use

$$
\frac{L_{n \overrightarrow{1}}^{\prime}(x)}{L_{n \overrightarrow{1}}(x)}=\sum_{k=0}^{n-1}\left(\frac{L_{(k+1) \overrightarrow{1}}^{\prime}(x)}{L_{(k+1) \overrightarrow{1}}(x)}-\frac{L_{k \overrightarrow{1}}^{\prime}(x)}{L_{k \overrightarrow{1}}(x)}\right),
$$

where $k \overrightarrow{1}=(k, k, \ldots, k)$ and $(k+1) \overrightarrow{1}=(k+1, k+1, \ldots, k+1)$. However, because of the scaling, we need to consider (observe that $|n \overrightarrow{1}|=r n$ )

$$
\frac{L_{n \overrightarrow{1}}^{\prime}(r n x)}{r n L_{n \overrightarrow{1}}(r n x)}=\sum_{k=0}^{n-1}\left(\frac{L_{(k+1) \overrightarrow{1}}^{\prime}(r n x)}{r n L_{(k+1) \overrightarrow{1}}(r n x)}-\frac{L_{k \overrightarrow{1}}^{\prime}(r n x)}{r n L_{k \overrightarrow{1}}(r n x)}\right),
$$

so that we can not use Cesàro's lemma to get the asymptotic behavior. We modify the proof as follows. For $\frac{k}{n} \leq t<\frac{k+1}{n}$ one has $\lfloor n t\rfloor=k$, hence the sum can be written as an integral

$$
\frac{L_{n \overrightarrow{1}}^{\prime}(r n x)}{r n L_{n \overrightarrow{1}}(r n x)}=n \int_{0}^{1}\left(\frac{L_{(\lfloor n t\rfloor+1) \overrightarrow{1}}^{\prime}(r n x)}{r n L_{(\lfloor n t\rfloor+1) \overrightarrow{1}}(r n x)}-\frac{L_{\lfloor n t\rfloor \overrightarrow{1}}^{\prime}(r n x)}{r n L_{\lfloor n t\rfloor \overrightarrow{1}}(r n x)}\right) d t,
$$

and the integrand can be written as

$$
\left(\frac{L_{(\lfloor n t\rfloor+1) \overrightarrow{1}}(r n x)}{r n L_{\lfloor n t\rfloor \overrightarrow{1}}(r n x)}\right)^{\prime} /\left(\frac{L_{(\lfloor n t\rfloor+1) \overrightarrow{1}}(r n x)}{r n L_{\lfloor n t\rfloor \overrightarrow{1}}(r n x)}\right) .
$$

So we need to know the asymptotic behavior of the ratio

$$
\lim _{n \rightarrow \infty} \frac{L_{(\lfloor n t\rfloor+1) \overrightarrow{1}}(r n x)}{r n L_{\lfloor n t\rfloor \overrightarrow{1}}(r n x)}
$$

If we change $n$ to $r n$ in Section 5 then for $q_{j} \rightarrow \frac{1}{r}(1 \leq j \leq r)$ we get the multi-index $n \overrightarrow{1}=(n, n, \ldots, n)$ and $(5.3)$ becomes

$$
\lim _{n \rightarrow \infty} \frac{L_{n \overrightarrow{1}+\vec{e}_{k}}(r n x)}{r n L_{n \overrightarrow{1}}(r n x)}=z(x)-\frac{r+1}{r},
$$

but we need to extend this for multi-indices containing the parameter $0<t \leq 1$. For this we need to use the following asymptotic behavior of the recurrence coefficients: if $\vec{n}=\left(\left\lfloor n q_{1}\right\rfloor,\left\lfloor n q_{2}\right\rfloor, \ldots,\left\lfloor n q_{r}\right\rfloor\right)$ and $\vec{m}=\left(\left\lfloor n t q_{1}\right\rfloor,\left\lfloor n t q_{2}\right\rfloor, \ldots,\left\lfloor n t q_{r}\right\rfloor\right)$, then

$$
\lim _{n \rightarrow \infty} \frac{a_{\vec{m}, j}}{n^{2}}=t^{2} q_{j}^{r+1} \prod_{i \neq j} \frac{1}{q_{j}-q_{i}}=t^{2} a_{j}, \quad 1 \leq j \leq r,
$$

and

$$
\lim _{n \rightarrow \infty} \frac{b_{\vec{m}, j}}{n}=t\left(1+q_{j}\right)=t b_{j}, \quad 1 \leq j \leq r .
$$

The required asymptotic behavior is then for $0<t \leq 1$

$$
\lim _{n \rightarrow \infty} \frac{L_{\vec{m}+\vec{e}_{k}}(n x)}{n L_{\vec{m}}(n x)}=z(x, t)-t b_{k},
$$


uniformly for $x$ on compact subsets of $\mathbb{C} \backslash[0, \infty)$, where $z(x, t)$ satisfies the algebraic equation

$$
(z(x, t)-x) B_{r}(z, t)+A_{r-1}(z, t)=0,
$$

with $B_{r}(z, t)=\prod_{j=1}^{r}\left(z-t b_{j}\right)=t^{r} B_{r}(z / t)$ and

$$
\frac{A_{r-1}(z, t)}{B_{r}(z, t)}=\sum_{j=1}^{r} \frac{t^{2} a_{j}}{z-t b_{j}}
$$

so that $A_{r-1}(z, t)=t^{r+1} A_{r-1}(z / t)$. Here we used $A_{r-1}(z)=A_{r-1}(z, 1)$ and $B_{r}(z)=$ $B_{r}(z, 1)$, which are the polynomials in Section 5. If $q_{j} \rightarrow \frac{1}{r}(1 \leq j \leq r)$ then $b_{j} \rightarrow \frac{r+1}{r}$ $(1 \leq j \leq r)$ and the algebraic equation for $z(x, t)$ becomes

$$
x\left(z(x, t)-t \frac{r+1}{r}\right)^{r}=(z(x, t)-t)^{r+1} .
$$

Now change $n$ to $r n$ so that we can deal with the multi-index $n \overrightarrow{1}=(n, n, \ldots, n)$. By going from the multi-index $n \overrightarrow{1}=(n, n, \ldots, n)$ to $(n+1) \overrightarrow{1}=(n+1, n+1, \ldots, n+1)$ in $r$ steps (each time increasing one coefficient) we then get

$$
\lim _{n \rightarrow \infty} \frac{L_{(\lfloor n t\rfloor+1) \overrightarrow{1}}(r n x)}{(r n)^{r} L_{\lfloor n t\rfloor \overrightarrow{1}}(r n x)}=\left(z(x, t)-t \frac{r+1}{r}\right)^{r},
$$

so that

$$
\lim _{n \rightarrow \infty} \frac{1}{r n} \frac{L_{n \overrightarrow{1}}^{\prime}(r n x)}{r n L_{n \overrightarrow{1}}(r n x)}=\frac{1}{r} \int_{0}^{1} \frac{\frac{d}{d x}\left(z(x, t)-t \frac{r+1}{r}\right)^{r}}{\left(z(x, t)-t \frac{r+1}{r}\right)^{r}} d t=\int_{0}^{1} \frac{z^{\prime}(x, t)}{z(x, t)-t \frac{r+1}{r}} d t,
$$

uniformly on compact subsets of $\mathbb{C} \backslash[0, \infty)$, where the prime' means the derivative with respect to $x$. This limit is the Stieltjes transform of the asymptotic zero distribution

$$
\int_{0}^{c_{r} / r} r \frac{u_{r}(r s)}{x-s} d s=\int_{0}^{c_{r}} \frac{u_{r}(y)}{x-y / r} d y
$$

and hence we have

$$
\int_{0}^{1} \frac{z^{\prime}(x, t)}{z(x, t)-t \frac{r+1}{r}} d t=\int_{0}^{c_{r}} \frac{u_{r}(y)}{x-y / r} d y .
$$

Observe that the change of variables $r z=t \hat{z}$ and $r x=t \hat{x}$ transforms the algebraic equation (6.2) to (3.1), so that $z(t \hat{x} / r, t)=t \hat{z}(\hat{x}) / r$. From our analysis in Sections 24 we found that

$$
\frac{\hat{z}^{\prime}}{\hat{z}-r-1}=\int_{0}^{c_{r}} \frac{w_{r}(s)}{\hat{x}-s} d s
$$

hence

Therefore

$$
\frac{z^{\prime}(x, t)}{z(x, t)-t \frac{r+1}{r}}=\frac{r}{t} \int_{0}^{c_{r}} \frac{w_{r}(s)}{\frac{r x}{t}-s} d s=\int_{0}^{c_{r}} \frac{w_{r}(s)}{x-\frac{t s}{r}} d s .
$$

$$
\begin{aligned}
\int_{0}^{1} \frac{z^{\prime}(x, t)}{z(x, t)-t \frac{r+1}{r}} d t & =\int_{0}^{1} \int_{0}^{c_{r}} \frac{w_{r}(s)}{x-\frac{t s}{r}} d s d t \\
& =\int_{0}^{1} \int_{0}^{t_{r}} \frac{w_{r}(y / t)}{x-\frac{y}{r}} \frac{d y}{t} d t \\
& =\int_{0}^{c_{r}} \frac{1}{x-\frac{y}{r}} \int_{y / c_{r}}^{1} w_{r}(y / t) \frac{d t}{t} d y
\end{aligned}
$$


where we used the change of variables $t s=y$ in the second equality and Fubini's theorem for the third equality. This means that

$$
u_{r}(y)=\int_{y / c_{r}}^{1} w_{r}(y / t) \frac{d t}{t}=\int_{y}^{c_{r}} w_{r}(x) \frac{d x}{x},
$$

and hence the asymptotic density of the scaled zeros $u_{r}$ is the Mellin convolution of the density $w_{r}$ given in (1.4) and the uniform distribution on $[0,1]$. This immediately gives the moments

$$
\int_{0}^{c_{r}} y^{n} u_{r}(y) d y=\int_{0}^{c_{r}} x^{n} w_{r}(x) d x \int_{0}^{1} t^{n} d t=\frac{1}{n+1}\left(\begin{array}{c}
(r+1) n \\
n
\end{array}\right) .
$$

We still need to show that the density $u_{r}$ is given by the expression in (1.5). Observe that the derivative of (1.5) with respect to $\varphi$ is

$$
\frac{r+1}{\pi} \frac{\sin \varphi(\sin r \varphi)^{r}}{(\sin (r+1) \varphi)^{r+1}}=\frac{r+1}{\pi \hat{x}} .
$$

On the other hand, taking the derivative in (6.3) with respect to $\varphi$ gives

$$
\frac{d u_{r}(\hat{x})}{d \varphi}=-\frac{w_{r}(\hat{x})}{\hat{x}} \hat{x}^{\prime}=\frac{r+1}{\pi \hat{x}},
$$

where we used (1.4) for the last equality. Thus, using $u_{r}\left(c_{r}\right)=0$, we find that

$$
u_{r}(\hat{x})=\frac{1}{r \pi} \frac{(\sin r \varphi)^{r+1}}{(\sin (r+1) \varphi)^{r}} .
$$

\section{Concluding remarks}

There is yet another family of multiple orthogonal polynomials for which the asymptotic distribution of the zeros is of the same flavor. These are multiple orthogonal polynomials associated with Meijer G-functions, which appear in the study of products of Ginibre random matrices [11]. The polynomials on the stepline for $|\vec{n}|=n$ are given by

$$
P_{n}(x)=(-1)^{n} \prod_{j=1}^{r}\left(n+\nu_{j}\right) ! \sum_{k=0}^{n}\left(\begin{array}{l}
n \\
k
\end{array}\right) \frac{(-x)^{k}}{\left(k+\nu_{1}\right) ! \cdots\left(k+\nu_{r}\right) !},
$$

and the asymptotic distribution of the scaled zeros $\left\{x_{k, n} / n^{r}, 1 \leq k \leq n\right\}$ is given in [16, Thm. 3.2]. The density is

$$
g_{r}(x)=\frac{1}{\pi} \frac{\sin ^{2} \varphi(\sin r \varphi)^{r-1}}{(\sin (r+1) \varphi)^{r}}
$$

where again

$$
x=\frac{(\sin (r+1) \varphi)^{r+1}}{\sin \varphi(\sin r \varphi)^{r}}, \quad 0<\varphi<\frac{\pi}{r+1} .
$$

This is the density of the Fuss-Catalan distribution, for which the moments are the FussCatalan numbers

$$
\int_{0}^{c_{r}} x^{n} g_{r}(x) d x=\frac{1}{r n+1}\left(\begin{array}{c}
(r+1) n \\
n
\end{array}\right), \quad n \in \mathbb{N} .
$$


Observe that the density $g_{r}$ is a Mellin convolution of the density $w_{r}$ in (1.4) and the $\operatorname{beta}\left(\frac{1}{r}, 1\right)$ density:

$$
\begin{aligned}
g_{r}(y) & =\frac{1}{r} \int_{y / c_{r}}^{1} w_{r}(y / t) t^{1 / r-1} \frac{d t}{t} \\
& =\frac{1}{r} \int_{y}^{c_{r}} w_{r}(x)\left(\frac{y}{x}\right)^{1 / r-1} \frac{d x}{x} \\
& =y^{1 / r-1} \frac{r+1}{r \pi} \int_{0}^{\theta} \frac{d \varphi}{x^{1 / r}}
\end{aligned}
$$

where

$$
y(\theta)=\frac{(\sin (r+1) \theta)^{r+1}}{\sin \theta(\sin r \theta)^{r}} .
$$

This can most easily be seen from

$$
\frac{d}{d \varphi}\left(\frac{(\sin \varphi)^{1 / r+1}}{(\sin (r+1) \varphi)^{1 / r}}\right)=\frac{r+1}{r} \frac{1}{x^{1 / r}}
$$

with $x$ given in (7.1), which enables a straightforward computation of the last integral in (7.2). The case $r=1$ corresponds to the asymptotic zero distribution of Laguerre polynomials (the Marchenko-Pastur distribution (1.6)). The case $r=2$ was obtained earlier in [3] and corresponds to multiple orthogonal polynomials for modified Bessel functions $K_{\nu}$ and $K_{\nu+1}$. The weight is then explicitly given by $g_{2}(x)=\frac{4}{27} h\left(\frac{4 x}{27}\right)$, where

$$
h(y)=\frac{3 \sqrt{3}}{4 \pi} \frac{(1+\sqrt{1-y})^{1 / 3}-(1-\sqrt{1-y})^{1 / 3}}{y^{2 / 3}}, \quad 0<y<1 .
$$

\section{Acknowledgements}

This research was supported by KU Leuven research grant OT/12/073, FWO research grant G.0934.13 and the Belgian Interuniversity Attraction Poles Programme P7/18. Thorsten Neuschel is a Research Associate (chargé de recherches) of FRS-FNRS (Belgian Fund for Scientific Research).

\section{References}

[1] M. Adler, P. van Moerbeke, D. Wang, Random matrix minor processes related to percolation theory, Random Matrices Theory Appl. 2 (2013), 1350008 (72 pages).

[2] A.I. Aptekarev, A. Branquinho, W. Van Assche, Multiple orthogonal polynomials for classical weights Trans. Amer. Math. Soc. 355 (2003), 3887-3914.

[3] E. Coussement, J. Coussement, W. Van Assche, Asymptotic zero distribution for a class of multiple orthogonal polynomials, Trans. Amer. Math. Soc. 360 (2008), no. $10,5571-5588$. 
[4] D. Dominici, W. Van Assche, Zero distribution of polynomials satisfying a differentialdifference equation, Anal. Appl. (Singap.) 12 (2014), no. 6, 635-666.

[5] P.J. Forrester, D.-Z. Liu, Raney distributions and random matrix theory, J. Stat. Phys. 158 (2015), 1051-1082.

[6] W. Gawronski, On the asymptotic distribution of the zeros of Hermite, Leguerre, and Joncquière polynomials, J. Approx. Theory 50 (1987), 214-231.

[7] R.L. Graham, D.E. Knuth, O. Patashnik, Concrete Mathematics, Addison-Wesley, Reading MA, 1989.

[8] M. Haneczok, W. Van Assche, Interlacing properties of zeros of multiple orthogonal polynomials, J. Math. Anal. Appl. 389 (2012), 429-438.

[9] M.E.H. Ismail, Classical and Quantum Orthogonal Polynomials in One Variable, Encyclopedia of Mathematics and its Applications 98, Cambridge University Press, 2005.

[10] A.B.J. Kuijlaars, Multiple orthogonal polynomial ensembles, in 'Recent Trends in Orthogonal Polynomials and Approximation Theory', Contemp. Math. 507, Amer. Math. Soc., Providence, RI, 2010, pp. 155-176.

[11] A.B.J. Kuijlaars, L. Zhang, Singular values of products of Ginibre random matrices, multiple orthogonal polynomials and hard edge scaling limits, Comm. Math. Phys. 332 (2014), no. 2, 759-781.

[12] V.A. Marchenko, L.A. Pastur, Distribution of eigenvalues for some sets of random matrices, Mat. Sb. 72 (114) (1967), no. 4, 507-536 (in Russian); translated in Math. USSR Sbornik 1 (1967), no. 4, 457-483.

[13] W. Młotkowski, K.A. Penson, K. Życzkowski, Densities of the Raney distributions, Doc. Math. 18 (2013), 1573-1596.

[14] E. Mukhin, A. Varchenko, Multiple orthogonal polynomials and a counterexample to the Gaudin Bethe Ansatz conjecture, Trans. Amer. Math. Soc. 359 (2007), no. 11, 5383-5418.

[15] K.A. Penson, K. Życzkowski, Product of Ginibre matrices: Fuss-Catalan and Raney distributions, Phys. Rev. E 83 (2011), 061118 (9 pages).

[16] T. Neuschel, Plancherel-Rotach formulae for average characteristic polynomials of products of Ginibre random matrices and the Fuss-Catalan distribution, Random Matrices Theory Appl. 3 (2014), no. 1, 1450003, 18 pp.

[17] L.R. Piñeiro Díaz, On simultaneous approximations for some collection of Markov functions, Vestnik Moskov. Univ. Ser. I Mat. Mekh. (1987), no. 2, 67-70 (in Russian); translated in Moscow Univ. Math. Bull. 42 (2) (1987), 52-55.

[18] G. Szegő, Orthogonal Polynomials, Amer. Math. Soc. Colloq. Publ. 23, Providence RI, 1939 (fourth edition 1975). 
[19] W. Van Assche, E. Coussement, Some classical multiple orthogonal polynomials, Numerical analysis 2000, Vol. V, Quadrature and orthogonal polynomials. J. Comput. Appl. Math. 127 (2001), 317-347.

[20] W. Van Assche, Nearest neighbor recurrence relations for multiple orthogonal polynomials, J. Approx. Theory 163 (2011), no. 10, 1427-1448.

[21] W. Van Assche, Ratio asymptotics for multiple orthogonal polynomials, Contemporary Mathematics (to appear); arXiv:1408.1829 [math.CA].

Walter Van Assche

Department of Mathematics

KU Leuven

Celestijnenlaan 200 B box 2400

BE-3001 Leuven

Belgium

walter@wis.kuleuven.be

Thorsten Neuschel

current address:

IRMP

Université Catholique de Louvain

Chemin du Cyclotron 2

BE-1348 Louvain-la-Neuve

Belgium

thorsten. neuschel@uclouvain. be 